\begin{tabular}{|c|l|}
\hline Title & The effect of tibial rotation on the presence of instability in the anterior cruciate ligament deficient knee. \\
\hline Author(s) & Samukawa, Mina; Magee, David; Katay ose, Masaki \\
\hline Citation & Journal of Sport Rehabilitation, 16(1), 2-17 \\
\hline Issue Date & $2007-02$ \\
\hline Doc URL & http://hdl.handle.net/2115/44329 \\
\hline Type & article \\
\hline File Information & JSR(Samukawa).pdf \\
\hline
\end{tabular}

Instructions for use 
ORIGINAL RESEARCH REPORTS

J Sport Rehabil. 2007,16, 2-17

(C) 2007 Human Kinetics, Inc.

\title{
The Effect of Tibial Rotation on the Presence of Instability in the Anterior Cruciate Ligament Deficient Knee
}

\author{
Mina Samukawa, David Magee, \\ and Masaki Katayose
}

\begin{abstract}
Context: The effects of tibial rotation after ACL injury have not yet been well determined. Objective: To show whether clinical outcomes such as the amount of tibial rotation can affect functional outcomes in normal and ACL deficient knees. Design: Case control study. Setting: Research laboratory. Participants: Twenty normal subjects (Control) and 20 subjects with ACL deficient knees (ACL). Main Outcome Measures: Tibial rotation at 30 and 90 degrees of knee flexion was measured using an inclinometer. One-legged hop, crossover hop, figure-of-eight running and 10-m running tests were used and determined the effect(s) of tibial rotation on the outcome of the functional tests. Results: There were significant between-group differences in internal and external rotation. The relationship between external tibial rotation and the figure-of-eight index was significantly negatively correlated. Conclusions: The amount of tibial rotation is greater in ACL ruptured knees than in uninjured knees, and these greater amounts of tibial rotation affected the figure-of-eight running index. Key Words: tibia rotation, ACL, functional test
\end{abstract}

The ligaments of the knee along with its surrounding muscles together play a crucial role in controlling the displacement of the tibia on the femur. The knee joint itself is able to move flexion-extension, varus-valgus, and internal rotationexternal rotation. ${ }^{1}$ Tibial rotation is an important element of physiological knee function and a common component of many knee injuries. ${ }^{2}$

The primary function of the ACL is to limit the anterior translation of the tibia relative to the femur, but it also plays a role in governing coupled tibiofemoral axial rotation with knee flexion. ${ }^{3}$ The effect on tibial rotation of cutting the ACL has been well reported. ${ }^{4,5,6,7}$ In a cadaveric study, ${ }^{4}$ total (internal and external) rotation at 10 and 30 degrees of knee flexion increased significantly after the ACL was cut. Another study done using biplanar photography ${ }^{8}$ showed that the ACL prevents internal rotation. Sectioning the ACL, other ligaments and structures significantly

Mina Samukawa is with the Department of Physical Therapy, Faculty of Health Sciences at Hokkaido University School of Medicine, Japan. E-mail: mina@cme.hokudai.ac.jp. David Magee is with the Department of Physical Therapy, University of Alberta, Canada. Masaki Katayose is with the Department of Physical Therapy, Graduate School of Health Sciences, Sapporo Medical University, Japan. 
increased tibial rotation. ${ }^{9,10}$ Therefore, severing the ACL greatly increases the amount of tibial rotation. On the other hand, a study that compared ACL injured knees with normal knees by using a Cybex dynamometer ${ }^{4}$ to measure rotational motion showed injured knees had significantly greater external and internal rotation compared to normal knees $(P<0.05)$. The test-retest reliability for internal rotation was relatively low in this study, however $(\mathrm{ICC}=0.34-0.59)$, compared with external rotation $(\mathrm{ICC}=0.84-1.0)$.

An assessment of knee rotation during dynamic activity is also important in relation to the functional stability of the knee. Significant increases in tibiofemoral rotation accompanied increased walking and running speeds in ACL injured knees. ${ }^{11}$ A separate study on the gait pattern of patients with ACL deficient knees reported that the tibia rotated internally during the initial swing phase, whereas in normal knees it rotated externally. ${ }^{12}$

Functional tests, which are designed to simulate the stress the knee encounters during selected functional activities, are both useful and simple to perform and are commonly used to determine such functional limitations as running and hopping in patients with ACL injuries. ${ }^{5,13,14}$ Although unable to detect specific knee abnormalities, functional tests can be used to assess knee function in various clinical settings. ${ }^{15,16}$

Although ACL injuries are widely known to affect tibial rotation, the amount of rotation has not been well reported. The extent to which functional activities are affected following ACL injuries, as well as whether the amount of tibial rotation is related to the dynamic function of patients with ACL deficiencies both remain unclear. As such, the first purpose of this study was to determine and compare the amount of internal and external rotation of the tibia on the femur, in both normal and ACL injured individuals. Its second purpose was to report on the effects of tibial rotation in subjects with ACL injured knees as they performed functional tests.

\section{Materials and Methods}

\section{Subjects}

Twenty subjects (10 male and 10 female), all of who had sustained unilateral ACL injuries, volunteered for this study (ACL group). The mean age was $22.9 \pm 5.7$ years (range 16-35 years), the mean height was $166.7 \pm 8.8 \mathrm{~cm}$ (range $152-184 \mathrm{~cm}$ ), and the mean weight was $61.3 \pm 10.7 \mathrm{~kg}$ (range 46-84 kg). Activity levels were 7 competitive and 13 recreational. Fifteen had injuries to the right knee and 5 to the left knee. The mean time suffering from the ACL injury was $8.9 \pm 4.7$ (range 1-19) months. All the injuries were sustained while playing sports. Nineteen of the subjects were dominant on the right side, ie, they preferred to kick a ball with their right foot, and 1 on the left side (left-footed). Sixteen patients were injured on their dominant side. The criteria for inclusion in the ACL group included a complete ACL rupture diagnosis confirmed by arthroscopy or MRI, no other ligament or meniscus injuries, and active sports participation at the time of data collection. Subjects were excluded from the study if they had any swelling in the injured knee, any difficulties running or jumping, any injuries to the contralateral knee, or had injured the knee less than 1 month previously. The subjects were recruited 
from Matsuda Orthopaedic Hospital and Nippon Telegraph and Telephone (NTT) Corporation Hospital in Sapporo, Japan. All subjects demonstrated a full range of motion and had no effusion at the time of testing.

Twenty uninjured subjects (10 male and 10 female) volunteered for this study as a control group. The subjects had no history of neurological or musculoskeletal injury. They were matched for age, sports, and activity levels with the patients in the ACL group. The mean age was $21.6 \pm 4.9$ years (range 16-35 years), the mean height was $166.7 \pm 6.6 \mathrm{~cm}$ (range $155-175 \mathrm{~cm}$ ), and the mean weight was $63.4 \pm$ $10.4 \mathrm{~kg}$ (range $49-85 \mathrm{~kg}$ ). Activity levels were 5 competitive and 15 recreational. All subjects were dominant on the right side. There were no significant differences in age, height, weight, or activity levels between the 2 groups. All subjects agreed to participate in this study, having read the information letter and signed an informed consent. The ethical committee of Sapporo Medical University approved this study.

\section{Measurement of Internal and External Tibial Rotation}

The amount of tibial rotation was measured using a Cybex EDI 320 inclinometer (Cybex Inc, Ronkonkowa, New York). This instrument is a portable electronic digital inclinometer that measures angular movements (Figure 1). Before each measurement, the inclinometer was calibrated by measuring standard constant angles.

Tibial rotation was measured at 30 degrees and 90 degrees of knee flexion. Subjects were placed in a supine position with hips flexed at 45 degrees. The hip and

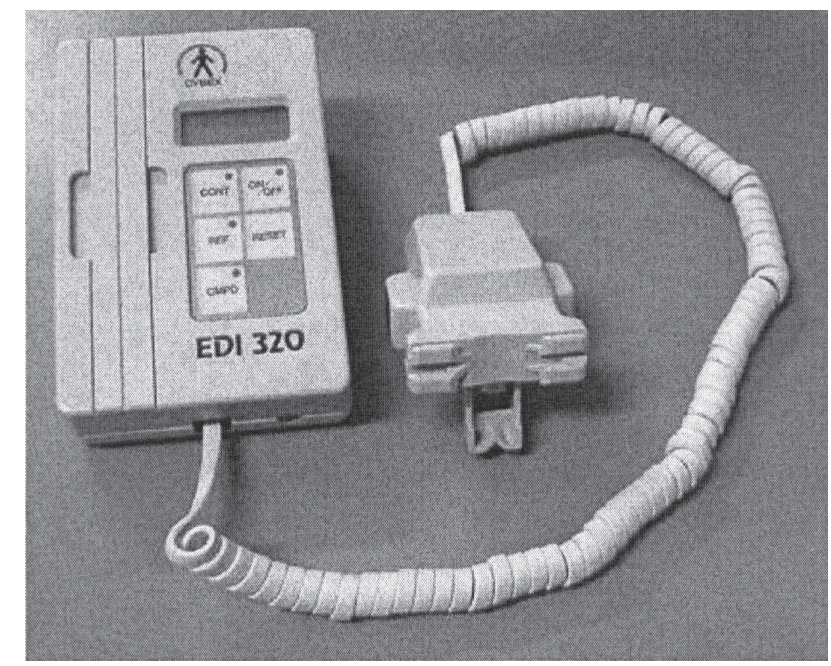

Figure 1-CYBEX EDI 320 inclinometer. 
knee angles were determined using a universal goniometer. The leg of each subject was placed in a specifically designed apparatus, which allowed the examiner to set the degree of knee flexion and to stabilize the femur and tibia. The inclinometer was stabilized on the leg using a purpose-built caliper made of wood to which the inclinometer had been firmly attached with a securing bolt (Figure 2). A rubber strap was also wrapped around the calf so the inclinometer was firmly secured to the center of the tibial tubercle. The neutral tibial rotation position was obtained by aligning the center of the tibial tubercle with the long axis of the femur. By grasping the tibia above the ankle joint, the tester passively rotated the tibia internally or externally until a tissue-stretch end feel was met and the motion stopped. The order of the measurement angles of knee flexion was set at random. All measurements were performed by one physical therapist, and to minimize measurement bias, internal or external rotation measurements were conducted alternately so that the tester would have greater difficulty remembering previous values. After taking each measurement, the tester read the value. The internal and external rotation tests were repeated three times, and the average value was used as the accepted value.

Before data collection, the intrarater reliability of the tibial rotation measurements collected using the inclinometer was determined. The measurements were performed on three normal female subjects (average age 32.3 years). The measurements were performed twice each at 30 and 90 degrees of knee flexion respectively, and the intraclass correlations were then calculated. The intrarater reliability of the tibial rotation measurements were as follows: ICC of internal rotation was 0.97 and ICC of external rotation was 0.93 at 30 degrees of knee flexion; and ICC of internal rotation was 0.97 and ICC of external rotation 1.00, respectively, at 90 degrees of knee flexion. Reliability was deemed to be very high.

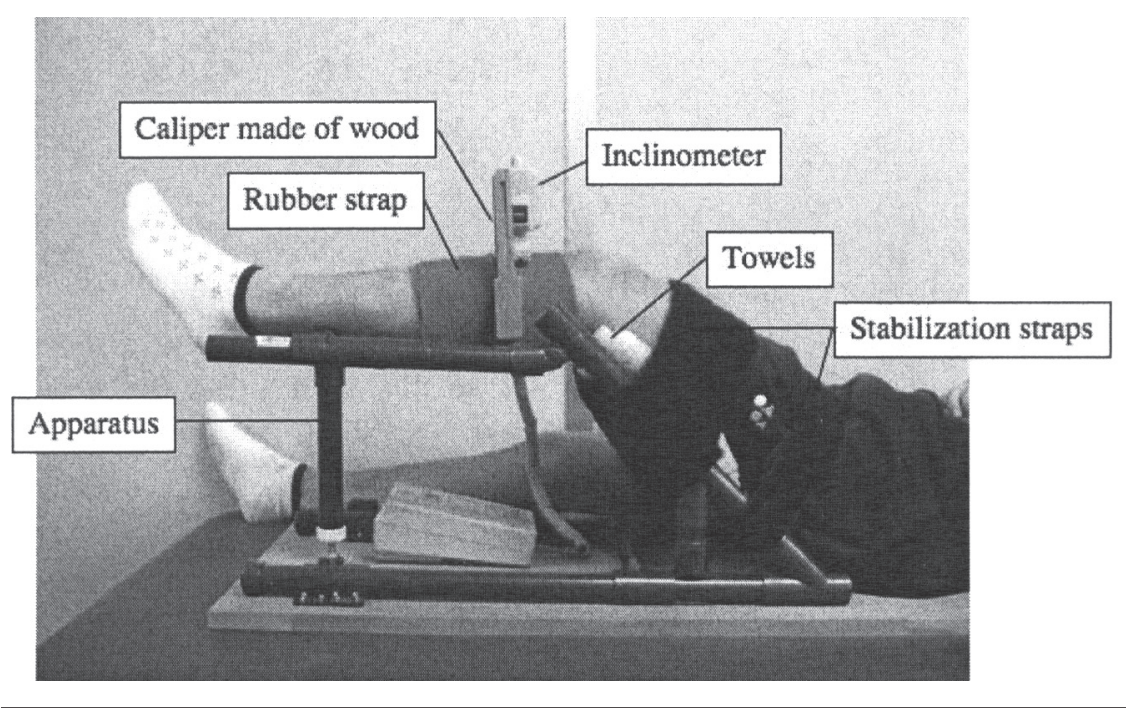

Figure 2-Position of subject when measuring tibial rotation. 


\section{Functional Tests}

Once the tibial rotation measurements were completed, each subject was asked to perform functional tests. Functional tests of the knee need to be sensitive to functional demands, so running, jumping, and twisting activities must be included. ${ }^{16}$ Previous studies recommended that at least 2 functional tests, such as the one-legged hop test and the crossover hop test, be conducted, as two tests were likely to find more abnormal scores for ACL deficient knees than one test alone. ${ }^{13}$ Another study reported that the figure-of-eight run test ratio (ie, figure-of-eight running time divided by $10-\mathrm{m}$ straight running time) was the best test for distinguishing normal and ACL deficient knees. ${ }^{17}$ Moreover, the one-legged hop test was used to determine the effect of straight forward jumping. The crossover hop test was utilized to assess the effect of side-to-side and cutting movements. The figure-of-eight test was also selected, as it comprised the movements of running and changing direction and included a rotational motion at the knee. Therefore, the one-legged hop test, crossover hop test, and figure-of-eight run test were chosen for the present study (Figure 3). All subjects performed the tests without braces or taping. A 10-minute run was given as a warm-up before the functional tests began, and subjects were told they could stop or refuse at any time if they felt afraid, unstable, or uncomfortable. To prevent any possible learning effects, all functional tests were repeated twice on the same day without any practice time allocated. ${ }^{15}$

The one-legged hop test ${ }^{13}$ was performed with the subject standing on one leg. The subject was asked to hop once as far as possible, land on the same leg, and maintain his/her balance. Each subject began the test by standing on the dominant leg (Control group) or the uninjured leg (ACL group) with toes lined up at the zero point of a tape measure. The recorded measurement was the distance from the 0 point to the place where the back of the subject's heel hit the ground after a single

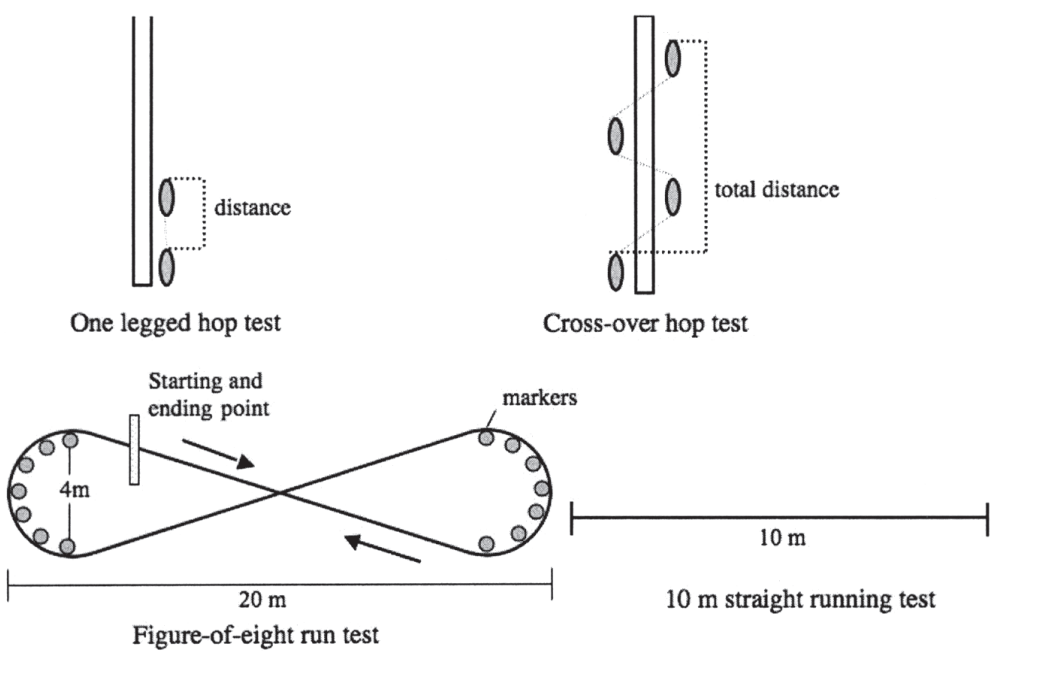

Figure 3-Functional tests. 
hop. The first leg was tested twice and the other leg was then tested. The average of the two values was used as the accepted value.

The crossover hop test ${ }^{13}$ was performed on a course consisting of a $15 \mathrm{~cm}$ wide marking strip on the floor, which extended for 6 meters. The subject hopped as far as possible 3 consecutive times on one leg, crossing over the center strip on each hop. Each subject started the test by standing on the dominant leg (Control group) and on the uninjured leg (ACL group) with the toes lined up at the zero point of the tape measure. The total distance of the hop was measured from the zero point to the place where the back of the subject's heel hit the ground after the third hop while maintaining his/her balance. The average of these values was used as the accepted value.

The figure-of-eight running test ${ }^{17}$ was measured using a 20 -m figure-of-eight running track with the diameter of both curves equaling 4 meters. The track was equipped with markers to prevent the subjects from performing overly short diameter turns. The starting point was located 2 meters from the beginning of the straight lines of the track. Photoelectronic cells (Digitimer Model: TW-7010A. Takei Kiki Kogyo CO. LTD) were placed 1 meter apart at the beginning of the straight line. The subjects were asked to run the figure eight course as fast as possible. The average of these values was used as the accepted value. The ten meter running time was also measured to obtain the figure-of-eight index described by Fonseca et al. ${ }^{17}$

Symmetrical indices (namely, the variables for the injured knees divided by the variables for the normal knees) were calculated for the following functional tests: the one-legged hop test values and the crossover hop test values. The data of the ACL group were compared to that of the Control group. The figure-of-eight index was obtained by calculating the figure-of-eight running time divided by 10-m running time.

\section{Data Analysis}

An unpaired $t$-test was used to calculate the demographic data (ie, age, height, and weight) to determine if any significant differences existed between the 2 groups. Differences in activity level, occupation, and dominant leg between the 2 groups were determined using the nonparametric Mann-Whitney's U test. Repeated analysis of variance $(2 \times 2)$ was used to determine the difference in the amount of internal and external tibial rotation between the Control group (right and left leg) and the ACL group (intact and injured side). Tukey's post-hoc test was used to examine any significant findings. The unpaired $t$-test was used to compare the results of the functional tests between the Control group and the ACL group. Pearson's Correlation Coefficient was used to describe the relationship between the amount of tibial rotation and the results of the functional tests. All analyses were performed using Statview 5.0 software (SAS Institutes Inc, Berkley, CA). Any $P$-value less than 0.05 was deemed significant.

\section{Results}

\section{Amount of Tibial Rotation}

The results for the amount of tibial rotation on the femur in the Control and ACL groups are presented in Table 1 . At both 30 degrees and 90 degrees of knee flex- 
ion, there was significantly greater internal tibial rotation in the injured leg of the ACL group than in the right and left legs in the Control group $(P<0.0001 \sim 0.004)$ or the intact side in the ACL group $(P<0.0001 \sim 0.0004)$. At 30 degrees of knee flexion the external tibial rotation of injured leg in the ACL group was significantly greater than in the right and left legs of the Control group $(P=0.0009 \sim 0.0080)$, or the intact side in the ACL group $(P=0.0200)$ as shown in Figure 4. At 90 degrees of knee flexion, the external tibial rotation of injured leg in the ACL group was significantly greater than in the right leg of the Control group $(P=0.021)$ as shown in Figure 5. However, there were no other significant differences of external tibial rotation at 90 degrees of knee flexion $(P=0.157 \sim 0.971)$. External rotation of the injured leg in the ACL group and the right leg in the Control group were determined significantly different $(P=0.0210)$. In the Control group, there were no significant differences for internal rotation at 30 or 90 degrees, or for external rotation at 30 degrees of knee flexion between the right and left legs, but there was a significant difference for external rotation between the left and right legs at 90 degrees of knee flexion $(P=0.0231)$.

\section{Functional Tests}

There were significant differences between the Control and ACL groups in both the one-legged hop $(P=0.013)$ and the crossover hop $(P=0.013$; Table 2$)$. However, the figure-of-eight index was not significantly different.

\section{Correlation Between the Amount of Tibial Rotation and the Functional Tests}

The results of correlation tests between the amount of tibial rotation and the functional tests in the ACL group are shown in Table 3. The amount of external rotation of the tibia on the femur and the results of the figure-of-eight test showed a moderate, negative correlation and were significantly different $(r=-0.464$ and $P=0.038$ at 30 degrees; $r=-0.542$ and $P=0.012$ at 90 degrees $)$.

\section{Comments}

Although the term "tibial rotation" has been used for many years, the question of how much internal and external rotation occurs has not been adequately answered. Several studies have been conducted to determine the amount of rotation, ${ }^{4,6}$ but most of the values reported were derived from cadaveric models. ${ }^{7,8,10,18}$ Furthermore, tibial rotation has been measured by inclinometers, electrogoniometers, video motion analyses, and roentogenograms, but all of these measurement devices, with the exception of the inclinometer, are expensive and the results are difficult to apply clinically. As such, an inclinometer was used in this study. Tibiofemoral rotation during functional movements such as walking, running, and cutting has been described using dynamic motion analysis, but the relationship between tibial rotation and functional tests is still unclear. It is important to develop a greater understanding of the relationship between tibial rotation and functional tests, which may be used to simulate a sports setting. 


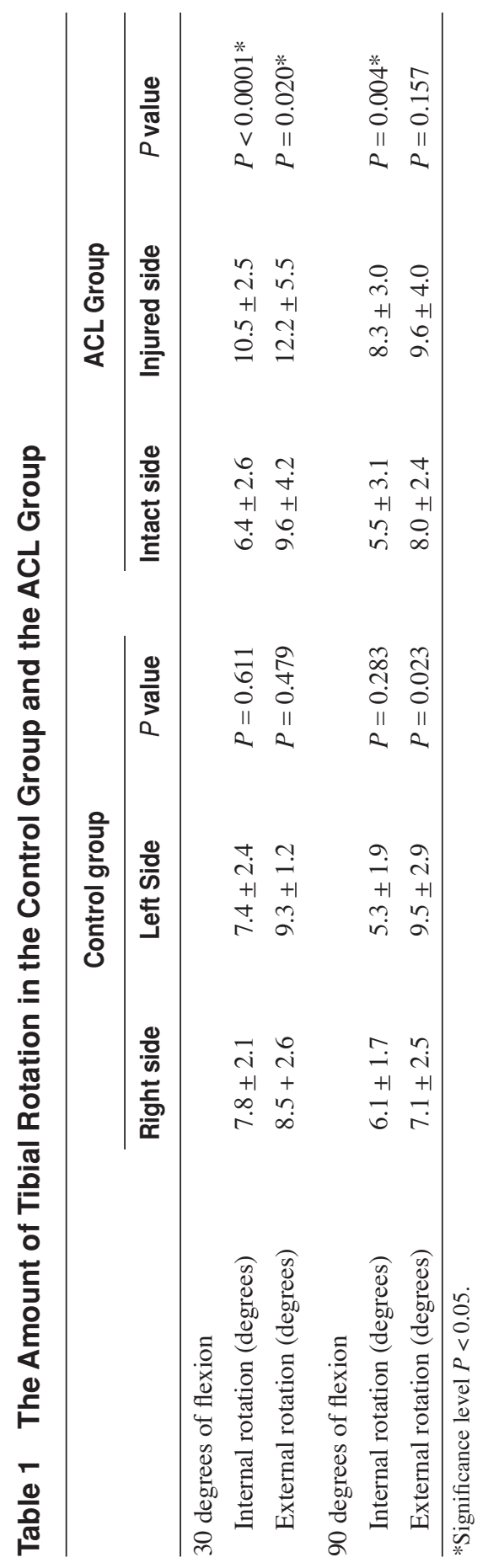


degrees

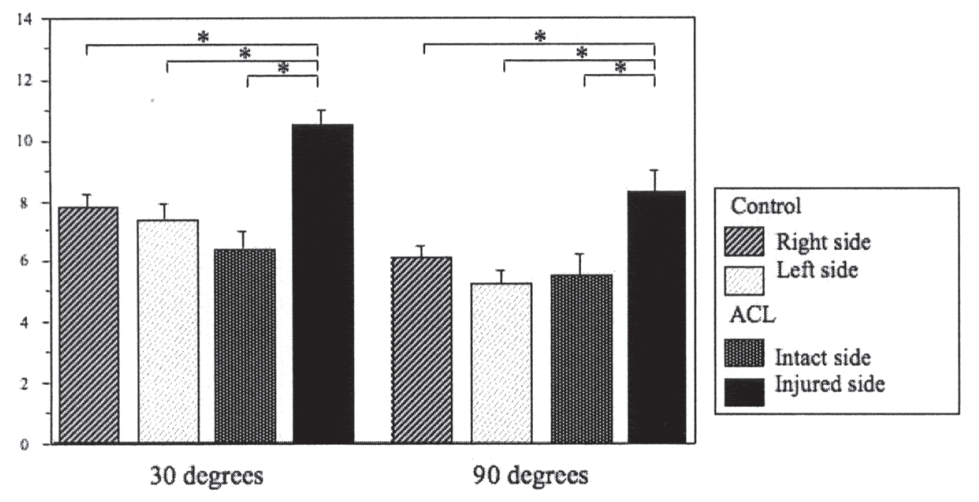

Figure 4-The amount of internal tibial rotation at 30 and 90 degrees of knee flexion in the Control group and the ACL group. The asterisk indicates significance at $\mathrm{P}<.05$.

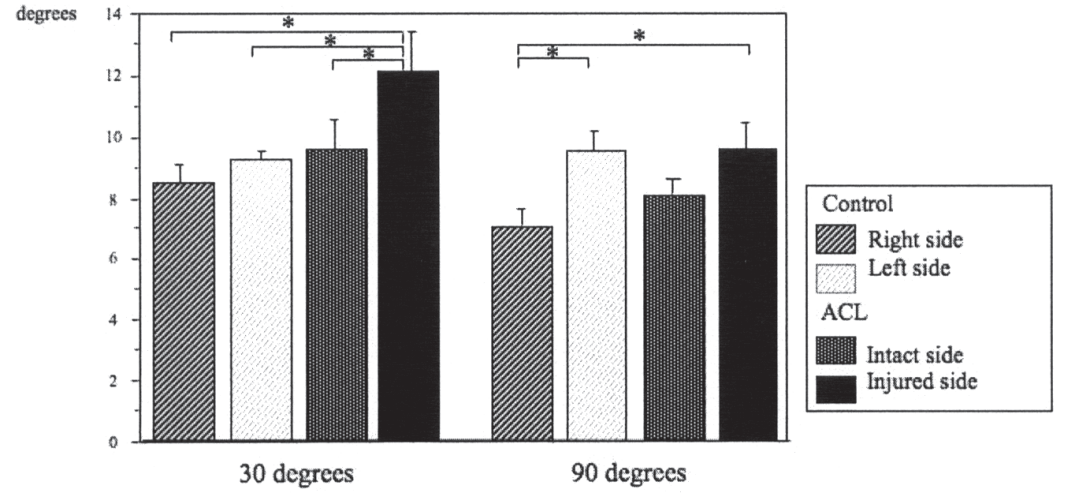

Figure 5-The amount of external tibial rotation at 30 and 90 degrees of knee flexion in the Control group and the ACL group. The asterisk indicates significance at $\mathrm{P}<.05$.

Table 2 Symmetrical Index Results of Functional Tests in the Control Group and the ACL Group

\begin{tabular}{lccc}
\hline & Control Group & ACL Group & $P$-Value \\
\hline One legged hop test & $1.01 \pm 0.08$ & $0.91 \pm 0.15$ & $P=0.013^{*}$ \\
Cross over hop test & $1.02 \pm 0.06$ & $0.87 \pm 0.24$ & $P=0.013^{*}$ \\
Figure-of-eight test & $7.98 \pm 0.63$ & $8.29 \pm 1.12$ & $P=0.295$ \\
\hline
\end{tabular}

*Significance level $P<0.05$. 
Tibial Rotation $\quad 11$

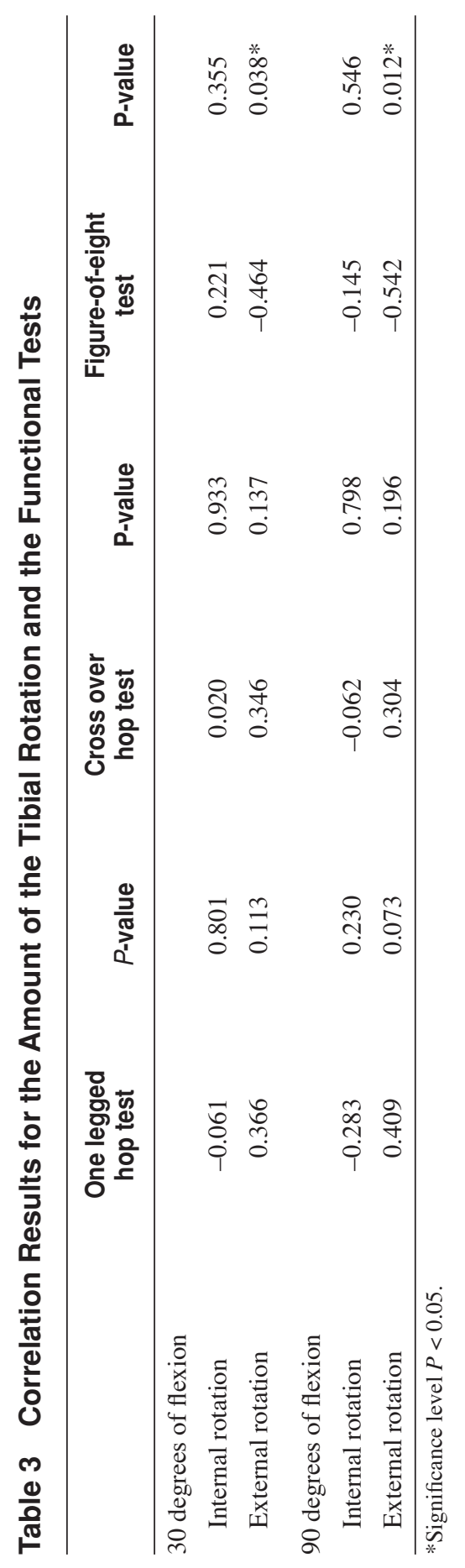




\section{Measurement of Tibial Rotation}

The amount of tibial rotation was hypothesized to be greater in ACL-ruptured knees than in normal (ie, uninjured) knees. In the present study, the amounts of internal tibial rotation at 30 degrees and 90 degrees of knee flexion, and of external tibial rotation at 30 degrees of knee flexion, were significantly larger in the ACL group than in the Control group $(P=0.0005-0.0284)$.

The primary function of the ACL is to prevent anterior translation of the tibia on the femur. A secondary role of the ACL is to resist tibial rotation, ${ }^{19}$ and it has been shown to restrain internal rotation more than external rotation. ${ }^{8}$ Zarins et $\mathrm{al}^{4}$ examined passive knee rotation in 17 subjects with normal knees and 19 patients with unilateral ACL injuries. They placed the subjects in a side lying position on a Cybex 2 isokinetic dynamometer, and measured rotation by securely attaching the subject's foot to the foot-plate of the dynamometer. Their results showed that ACL-torn knees had significantly more internal rotation at 15 degrees and more external and internal rotation at 5 degrees of knee flexion than the normal knees.

The present study showed that with the exception of external tibial rotation at 90 degrees, the amount of internal and external tibial rotation of the injured leg was significantly larger than the intact leg in the Control group and the right and left legs in the Control group $(P<0.05)$.

Cadaveric studies have also confirmed that the amount of tibial rotation was affected by ACL injury. Wroble et $\mathrm{al}^{9}$ examined tibiofemoral motion when lateral structures in the ACL deficient knees of 20 cadaveric lower limbs were sectioned and measured with a 6 degrees-of-freedom electrogoniometer. They found statistically significant increases in external rotation at all flexion angles when the ACL and lateral structures (ie, popliteus tendon, posterolateral capsule, and lateral collateral ligament) were cut. Matsumoto et $\mathrm{al}^{7}$ examined the rotational angles of the knee joint by severing the ACL and/or the MCL. Sections taken from 6 frozen knee specimens were measured using bi-planar photography. They found that once the ACL was cut, the amount of internal rotation increased. When the MCL was also severed, the external tibial rotation angle increased throughout the range of flexion (0-90 degrees). Both studies showed an increasing amount of rotation with knee flexion after the ACL and other structures were cut. At 90 degrees of knee flexion, other structures such as the MCL, LCL, the popliteus tendon, and the posterolateral capsule were thought to stabilize the knee joint. Subjects with isolated ACL injuries but no other injuries were chosen for this research, so no difference in external rotation at 90 degrees of knee flexion was shown between the 2 groups.

\section{Functional Tests}

Functional tests are designed to simulate the stress the knee encounters during physical activity. Functional tests cannot detect specific abnormalities, but are useful in assessing lower limb function. ${ }^{15}$ Many kinds of functional tests have been reported, but the reliability and validity of these tests are often unclear. Noyes et $\mathrm{al}^{13}$ recommended using at least two of the tests together when testing patients with ACL injuries because of the relatively low sensitivity scores of individual tests. Therefore, three functional tests were selected for this study. The results were determined using the symmetrical inde ${ }^{13}$ to ascertain the difference between normal and ACL injured groups. An assessment of possible rotational movements 
during dynamic activity was thought to be of more significance in relation to the functional stability of the knee.

It was felt that this combination of functional tests would have a greater effect on the ACL-ruptured knees of patients when compared with the knees of normal subjects. That is to say, subjects with normal legs would be able to jump further and do the tests faster than patients with injured legs.

The results of this study showed there were significant differences between the two groups in the symmetrical indices of the one-legged hop test $(P=0.0125)$ and the crossover hop test $(P=0.0134)$. Both tests demand coordinated muscular contractions to control the knee through the rapid deceleration during the first impact and through the ensuing rapid acceleration of straight- or cross-forward propulsion into the next jump. Episodes of the knee giving way in ACL-injured patients were most likely to occur during sporting activities that involve sudden stopping and cutting. ${ }^{20}$ These tests are thought to simulate the movements that might cause giving way and significant differences were shown between the groups. Therefore, tests such as one-legged hop and crossover hop are thought to be very useful for determining change following rehabilitation and evaluating the effects of ACL injury on performance.

The results of the figure-of-eight test in the present study, however, did not show significant differences between the two groups. The groups were compared using an index obtained by dividing the figure-of-eight running time by the 10meter straight running time. Fonseca et $\mathrm{al}^{17}$ reported that the figure-of-eight index was best for distinguishing the difference between normal and ACL deficient knees. However, the subjects taking part in the present study had already been participating in sporting activities prior to doing the tests so deceleration was thought to be less demanding for the figure-of-eight running test and the straight running test than for the hop test. Therefore, it is possible that the tests were not rigorous enough to detect differences between the 2 groups.

\section{Correlation Between the Amount of Tibial Rotation and Functional Tests}

Greater laxity of the knee might lead to greater difficulties in performing functional knee movements during sports. That is to say, clinical outcomes may affect functional outcomes. Anterior-posterior laxity measured by a KT-1000 arthrometer and the functional tests of patients with ruptured ACLs have been shown to be correlated, ${ }^{21,22}$ but this relationship is still controversial. The correlation between the amount of tibial rotation and the functional tests was also considered to be important because these correlations could show whether clinical outcomes such as the amount of tibial rotation can affect functional outcomes or not.

At the beginning of this study, it was felt that increased instability of the knee joint might lead to increase difficulties in performing functional knee movements. The results showed a significant negative correlation between the amount of external tibial rotation and the figure-of-eight index. That is to say, that greater amounts of tibial external rotation lead to lower figure-of-eight index values, meaning that both the figure-of-eight and 10-meter straight running times were longer in the ACL group. Aside from this significant negative correlation, however, there were no other statistically significant relationships between the amount of tibial rotation and the other functional tests. 
The relationship between the clinical outcomes of anterior laxity using a KT1000 or KT-2000 arthrometer and the functional outcomes has previously been described. ${ }^{7,22-25}$ Barber et al ${ }^{23}$ reported no significant relationships between arthrometric measurement (KT-1000) and five functional tests (ie, one-legged hop for distance, one-legged timed hop, one-legged vertical jump, shuttle run with pivot, and shuttle run without pivot). Fonseca et $\mathrm{al}^{17}$ found a significant positive correlation for knee ligament laxity, which was obtained using the KT-1000 arthrometer and the hop index (the distance hop for the normal leg divided by that of the injured leg). They also found that the ratio of figure-of-eight running to 10-meter straight running time was the best variable to differentiate ACL deficient knees from normal knees. However, the knee ligament laxity obtained by the KT-1000 was not correlated with the running tasks in their study. Neeb et $\mathrm{al}^{21}$ examined the association between clinical tests (Lachman test and pivot shift test), KT-1000, and functional tests (one-legged hop test and timed hop test) following ACL injuries. There were no significant associations (one-legged hop symmetry index versus KT-1000, $r=$ 0.19 ; timed hop symmetrical index versus KT-1000, $r=-0.02$ ), and they concluded that the low and insignificant associations could be explained by the fact that the clinical tests were directed at the impairment level, which determined loss or abnormality of physiological and anatomical structures, while the functional tests were directed at the disability level, which implies an inability due to the impact of pathology or insult on the functioning of specific body mechanisms. Eastlack et $\mathrm{al}^{22}$ determined the relationship among laxity, quadriceps strength, instability, and functional tests (ie, one-legged hop, crossover hop, and timed hop tests) between copers and noncopers suffering from ACL injuries. There was no significant difference in the anterior-posterior laxity of the knee between the copers and noncopers, but the functional test results of the copers were significantly better than those of the noncopers. Functional stability is provided by passive restraint from ligaments and joint geometry and by active restraint from muscles. These provide both static and dynamic stability. ${ }^{25}$ Therefore, active restraints such as muscles are thought to work in combination with passive restraints around the knee to limit motion between the tibia and the femur.

A biomechanical dynamic evaluation of tibiofemoral rotation in ACL-deficient knees during walking and running has also been conducted. ${ }^{11}$ A triaxial goniometer was used to test 11 patients with ACL ruptures and 9 normal subjects. Significant increases in the total tibiofemoral rotation were found during the stance phase of running compared with that of walking $(P<0.001)$, although there were no significant differences between the subjects with ACL injuries and normal subjects. There was a significant positive correlation between the isokinetic peak torque of quadriceps and hamstrings at 180 degrees/second and tibiofemoral rotation angle at higher running speeds, however. That is, the greater the isokinetic peak torque of quadriceps or hamstrings, the smaller the tibiofemoral rotation. Therefore, muscle strength may play a role in stabilizing rotational motion in the knee, and active restraints may prevent or decrease the occurrence of rotational instability.

In this study, significant negative correlations between external rotation and the figure-of-eight index were found. That is to say, greater amounts of tibial external rotation led to a lower time ratio for the figure-of-eight running test and the 10-meter straight running test. The greater the amount of external tibial rotation, the more 
time is needed to complete both figure-of-eight running and 10-m running tests or the more time is needed to complete the 10-m running test only. The figure-of-eight running test is designed for straight running, cutting, and changing direction with narrow turns. To do the test properly, acceleration and deceleration skills are also required. While cutting turns, deceleration and tibial rotation occur to maintain balance. ${ }^{26}$ Ten meter running test is needed to get the skill of dash. The dash includes acceleration so it seems difficult for the patients with ACL injured because of the possibilities to get giving way. ${ }^{20}$ Therefore, ACL injuries with the greater external tibial rotation might be affected by figure-of-eight running tests.

There were no significant correlations between the amount of tibial rotation and the other functional tests (ie, one-legged hop and crossover hop tests) in this study, however. All patients in the ACL group participated in sporting activities to some degree after injury. Noyes et $\mathrm{al}^{13}$ considered a symmetry index of 0.85 to be within the normal range. In the present study, the ACL-injured group showed an index of 0.91 for the one-legged hop test and 0.87 for the crossover hop test. The subjects with ACL injuries may have compensated by using dynamic stabilizers as active restraints. Another reason for the insignificant correlations between the amount of tibial rotation and the functional tests could be that the functional tests chosen were not sensitive enough to reveal any differences. Moreover, the subjects were wearing shoes while they were tested, which might mitigate the factors contributing to instability in ACL-injured patients by helping to maintain balance during the tests. ${ }^{27}$ Furthermore, some patients with ACL deficient knees were thought to cope well with knee instability even without surgical reconstruction. ${ }^{27}$

\section{Clinical Relevance of This Study}

During this study, an inclinometer was used to compare and determine the amount of tibial rotation in subjects with ACL-injured legs and normal (uninjured) legs. Results showed that tibial rotation was greater in the injured legs. The results of functional tests, such as the one-legged hop test and crossover hop test, were shown to be significantly different between the Control and the ACL groups. These tests can be used to determine the effect(s) of ACL injuries. The relationship between the external rotation of the tibia and the figure-of-eight index was significantly negatively correlated. That is to say, the effect of external tibial rotation was greatest when subjects were performing the figure-of-eight running test (greater time). Therefore, the patients who suffered an ACL injury with excessive amount of external tibial rotation might be affected during functional movements, which include running, cutting, and changing direction such as the figure-of-eight running test.

\section{Limitation of This Study}

The present study has several limitations. First, only subjects with isolated ACL injuries confirmed by arthroscopy or MRI and no meniscus injuries were chosen for this research. However, the subjects may have had other tissue injuries that could have affected the amount of tibial rotation. Previous studies using cadaveric knees have shown that the amount of tibial rotation changes after transection of the posterolateral complex and iliotibial band around the knees. ${ }^{7,10,20}$ 
Second, the amount of tibial rotation was only determined at 30 and 90 degrees of knee flexion in the present study. Cadaveric studies have also shown that the amount of tibia rotation changes at different angles of knee flexion. ${ }^{10,18,28-30}$

Third, although the functional tests showed several significant differences between the Control and the ACL groups, other functional tests such as the shuttle run test, which also includes running, stopping, and cutting maneuvers, may have a greater effect on the amount of the tibial rotation.

\section{Conclusion}

The amount of internal and external tibial rotation and 3 functional tests (ie, one-legged hop test, crossover cut test, and figure-of-eight running test) were compared in 20 normal subjects and 20 patients who had suffered ACL injuries. Internal tibial rotation at 30 degrees and 90 degrees of flexion and external rotation at 30 degrees of knee flexion in the injured side of ACL group showed significantly greater than the uninjured side of the ACL group and the both sides of Control group $(P<0.05)$. The one-legged hop test and the crossover hop test showed significant differences between the Control group and the ACL group $(P<0.05)$. The relationship between the amount of external rotation of the tibia and figure-of-eight test were significantly negatively correlated. Therefore, patients who suffered ACL injuries with an excessive amount of external tibial rotation might be affected by functional movements, such the figure-of-eight running test, which include running, cutting, and changing direction, provided the functional tests sufficiently stress the knee.

\section{References}

1. Takeda Y, Xerogeanes JW, Livesay GA, Fu FH, Woo SL: Biomechanical function of the human anterior cruciate ligament. Arthroscopy. 1994;10:140-147.

2. Shoemaker SC, Markolf KL: In vivo rotatory knee stability. Ligamentous and muscular contributions. J Bone joint Surg. 1982;64A:208-216.

3. Barrance PJ, Williams GN, Snyder-Mackler L, Buchanan TS. Altered knee kinematics in ACL-deficient non-copers: a comparison using dynamic MRI. J Orthop Res. 2006; 24:132-140.

4. Zarins B, Rowe CR, Harris BA, Watkins MP. Rotational motion of the knee. Am J Sports Med. 1983;11:152-156.

5. Andersen HN, Dyhre-Poulsen P. The anterior cruciate ligament does play a role in controlling axial rotation in the knee. Knee Surg Sports Traumatol Arthrosc. 1997;5:145-149.

6. Lusin, GF, Gajdosik RL. Reliability of instrumentation and measurement procedures for active internal and external rotation. J Orthop Sports Phys Ther. 1983;4:154-157.

7. Matsumoto H. Mechanism of the pivot shift. J Bone Joint Surg. 1990;72B:816-821.

8. Matsumoto H, Suda Y, Otani T, Niki Y, Seedhom BB. Roles of the anterior cruciate ligament and the medial collateral ligament in preventing valgus instability. J Ortho Sci. $2001 ; 6: 28-32$.

9. Wroble RR, Grood ES, Cummings JS, Henderson JM, Noyes FR. The role of the lateral extraarticular restraints in the anterior cruciate ligament-deficient knee. Am J Sports Med. 1993;21:257-262.

10. Veltri DM, Deng XH, Torzili PA, Maynard, MJ, Warren, RF. The role of the popliteofibular ligament in stability of the human knee: a biomechanical study. Am J Sports Med. 1996;24:19-27. 
11. Czerniecki JM, Lippert F, Olerud JE. A biomechanical evaluation of tibiofemoral rotation in anterior cruciate deficient knees during walking and running. Am J Sports Med. 1988;16:327-31.

12. Georgoulis AD, Papadonikolakis A, Papageorgiou CD, Mitsou A, Steriou N. Threedimensional tibiofemoral kinematics of the anterior cruciate ligament-deficient and reconstructed knee during walking. Am J Sports Med. 2003;31:75-79.

13. Noyes FR, Barber SD, Mangine RE. Abnormal lower limb symmetry determined by function hop tests after anterior cruciate ligament rupture. Am J Sports Med. 1991;19:513-518.

14. Itoh H, Kurosaka M, Yoshiya S, Ichihashi N, Mizuno K. Evaluation of functional deficits determined by four different hop tests in patients with anterior cruciate ligament deficiency. Knee Surg. Sports Traumatol, Arthrosc. 1998;6: 241-245.

15. Bolgla LA, Keskula DR. Reliability of lower extremity functional performance tests. J Orthop Sports Phys Therd. 1997;26:138-142.

16. Risberg MA, Ekeland HI. Reliability of functional knee tests in normal athletes. Scand J Med Sci Sports. 1995;5:24-28.

17. Fonseca ST, Magee DJ, Wessel J, Reid D. Validation of a performance test for outcome evaluation of knee function. Clin J Sport Med. 1992;2:251-256.

18. Kwak SD, Ahmad CS, Gardner TR et al. Hamstrings and iliotibial band forces affect knee kinematics and contact pattern. J Orthop Res. 2000;18:101-108.

19. Girgis FG, Marshall JL, Monajem A. The cruciate ligaments of the knee joint. Anatomical, functional and experimental analysis. Clin Orthop. 1975;106:216-231

20. Houck J, lerner A, Gushue D, Yack HJ. Self-reported giving-way episode during a stepping-down task: case report of a subject with an ACL-deficient knee. J Ortop Sports Phys Ther. 2003;273-281.

21. Neeb TB, Aufdemkampe G, Wagener JHD, Mastenbroek L. Assessing anterior cruciate ligament injuries: the association and differential value of questionnaires, clinical tests, and functional tests. J Orthop Sports Phys Ther. 1997;26:324-331.

22. Eastlack ME, Axe MJ, Snyder-Macker L. Laxity, instability, and functional outcome after ACL injury: copers and noncopers. Med Sci Sports Exerc. 1999;31:210-215.

23. Barber SD, Noyes FR, Mangine RE, McCloskey JW, Hartman W. Quantitative assessment of functional limitations in normal and anterior cruciate ligament-deficient knees. Clin Orthop. 1990;255:204-214.

24. Lephart SM, Perrin DH, Fu FH, Gieck JH, McCue FC, Irrgang JJ. Relationship between physical characteristics and functional capacity in the anterior cruciate ligament in sufficient athlete. J Orthop Sports Phys Ther. 1992;16:174-151.

25. Noyes FR, Grood ES, Butler DL, Malek M. Clinical laxity tests and functional stability of the knee: biomechanical concepts. Clin Orthop. 1980;146:84-9.

26. Houck J, Yack HJ. Associations of knee angles, moments and function among subjects that are healthy and anterior cruciate ligament deficient (ACLD) during straight ahead and crossover cutting activities. Gait and Postsure. 2003;18:126-138.

27. Ireland ML. Anterior cruciate ligament injury in female athletes: epidemiology. J Athl Train. 1999;34:150-154.

28. Matsumoto H, Seedhom BB, Suda Y, Otani T, Fujiwara K. Axis location of tibial rotation and its change with flexion angle. Clin Orthop. 2000;371:178-182.

29. Pasque C, Noyes FR, Gibbons M, Levy M, Grood E. The role of the popliteofibular ligament and the tendon of popliteus in providing stability in the human knee. $J$ Bone Joint Surg. 2003;85B:292-298.

30. Markolf KL, Mensch JS, Amstutz HC. Stiffness and laxity of the knee-the contributions of the supporting structures. A quantitative in vitro study. J Bone Joint Surg. 1976;58A:583-594. 